\title{
Production and Searches for Cascade Baryons with CLAS
}

\author{
Elton S. Smith* \\ for the CLAS Collaboration \\ Thomas Jefferson National Accelerator Facility, \\ 12000 Jefferson Avenue, Newport News, VA 23606, USA \\ *E-mail: elton@jlab.org
}

\begin{abstract}
We present the results of photoproduction cross sections of the ground state cascade $\Xi^{-}$and the first excited state $\Xi^{*-}$ (1530) measured with the CLAS detector. The photoproduction of the cascade resonances has been investigated in the reactions $\gamma p \rightarrow K^{+} K^{+}(X)$ and $\gamma p \rightarrow K^{+} K^{+} \pi^{-}(X)$. The differential and total cross sections of the $\Xi^{-}$were determined for photon beam energies from 2.75 to $4.75 \mathrm{GeV}$ and are consistent with a production mechanism of $Y^{*} \rightarrow K^{+} \Xi^{-}$through a t-channel process. The cross-section of the $\Xi^{*-}(1530)$ has been determined for photon beam energies from 3.35 to $4.75 \mathrm{GeV}$. The reaction $\gamma p \rightarrow K^{+} K^{+} \pi^{-}\left(\Xi^{0}\right)$ has also been investigated to search for excited cascade resonances decaying to $\pi^{-} \Xi^{0}$. No significant signal of excited cascade states other than the well-known $\Xi^{*-}(1530)$ is observed.

We also present the latest results of a search for the $\Phi^{--}(1862)$ exotic pentaquark state in a photoproduction experiment on a deuterium target. A high-statistics sample of $\pi^{-} \Xi^{-}$events have been collected and analyzed. A preliminary invariant mass spectrum of the $\pi^{-} \Xi^{-}$system is presented, which is used to set upper limits on the photoproduction of the $\Phi^{--}$pentaquark state.

Keywords: Cascade resonances; hyperon photoproduction; cascade pentaquarks
\end{abstract}

\section{Introduction}

The $\Xi$ resonances are largely under-explored when compared with the nonstrange baryons and $S=-1$ hyperon states. Only two ground state cascades, the octet member $\Xi$ and the decuplet member $\Xi(1530)$, have four stars assigned by the Particle Data Group (PDG), ${ }^{1}$ and four others have three-stars. This is mainly due to smaller cross sections for the $S=0$ and $S=-1$ baryons, and the fact that the cascade resonances cannot be produced through direct formation. More than $20 N^{*}$ and $\Delta^{*}$ resonances are rated with at least three stars in the PDG. Flavor $S U(3)$ sym- 


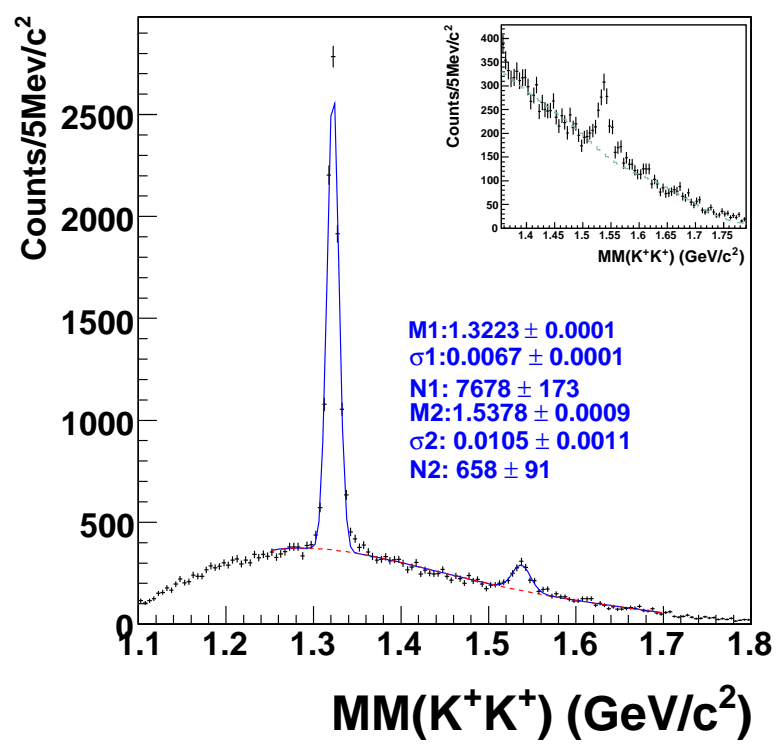

Fig. 1. $M M\left(K^{+} K^{+}\right)$distribution for $E_{\gamma}>2.6 \mathrm{GeV}$ fitted with two Gaussian functions and the empirical background shape with adjustable normalization ( $\mathrm{M}$ : mean of the Gaussian peak position, $\sigma$ : width of the Gaussian signal, N: number of events in the peak); Inset: $M M\left(K^{+} K^{+}\right)$distribution enlarged for the $1.36-1.79 \mathrm{GeV} / \mathrm{c}^{2}$ region, the dashed lines show the empirical background shape from $K^{-}$events normalized to the region of $1.36-1.5 \mathrm{GeV} / \mathrm{c}^{2}$.

metry predicts as many $\Xi$ resonances as $N^{*}$ and $\Delta^{*}$ states, suggesting that many more cascade resonances await to be discovered. Of the six established $\Xi$ resonances, only three have measured spin-parity $\left(J^{P}\right)$ assignments $\left(\Xi(1320) \frac{1}{2}^{+}, \Xi(1530) \frac{3}{2}^{+}, \Xi(1820) \frac{3}{2}^{-}\right)$.

The missing cascade states in the excitation spectrum motivates studies of cascade production and also raises the question whether the standard quark model is adequate to describe the nature of baryons with two strange quarks. It is therefore natural to search for cascade states that may occur outside the standard quark model. In this paper we first present measurements with the CLAS detector ${ }^{2}$ of the photoproduction of known cascade states, and then report on a search for exotic states. 


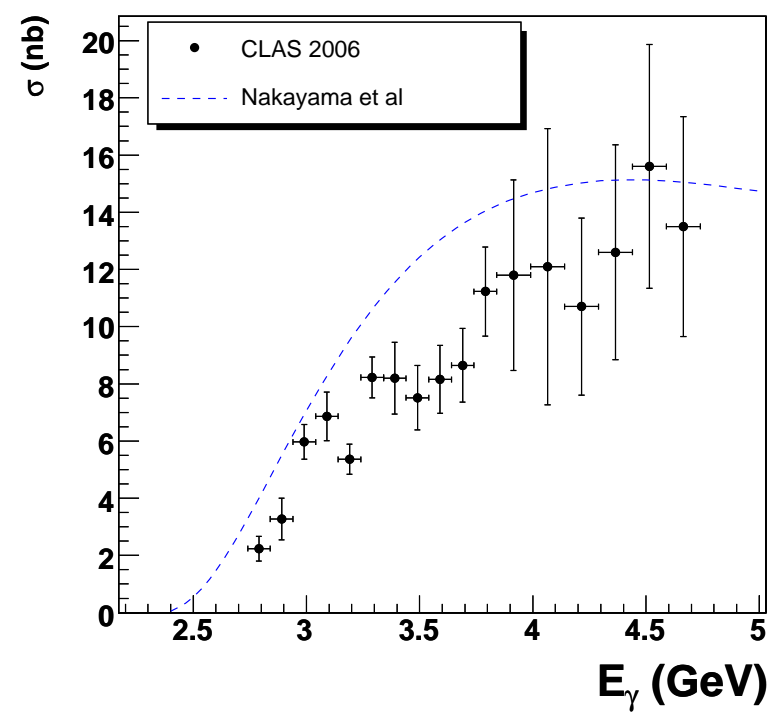

Fig. 2. Total cross section for the ground state $\Xi^{-}$is plotted as a function of photon energy $^{3}$ and compared with a model prediction. ${ }^{4}$

\section{Photoproduction of known cascade states}

The production cross sections of the $\Xi^{-}(1321)$ ground state and the excited $\Xi^{-}(1530)$ state $^{3}$ were determined from data registered during photon interactions with a proton in the CLAS detector, ${ }^{2}$ collecting an integrated luminosity of about $70 \mathrm{pb}^{-1}$. The primary electron beam energy, $E_{0}$, for most of the data sample was $4 \mathrm{GeV}$, which generated photons in the range between 1.6-3.85 GeV. A small amount of data were collected with $E_{0}=5 \mathrm{GeV}$, which extended the photon energy range to $4.75 \mathrm{GeV}$. The cascade resonances were identified via missing mass in the reaction $\gamma p \rightarrow K^{+} K^{+}(X)$ and in the reaction $\gamma p \rightarrow K^{+} K^{+} \pi^{-}(X)$ from the decay $\Xi^{*-} \rightarrow \Xi^{0} \pi^{-}$. The double-strangeness was tagged by detecting two positive kaons in CLAS, ${ }^{2}$ and the cascade resonances were observed in the $K^{+} K^{+}$missing mass spectrum (Fig. 1). The $\Xi^{-}$mass was determined to be $1322.3 \pm 0.1 \pm 1.2 \mathrm{MeV} / \mathrm{c}^{2}$. The measured width of $6.7 \pm 0.1 \mathrm{MeV} / \mathrm{c}^{2}$ is consistent with the expected missing mass resolution of CLAS. Approximately $7700 \Xi^{-}$events were identified for the photon energy range of 2.6 to $4.75 \mathrm{GeV}$.

These data represent the highest statistics collected for exclusive photoproduction of $\Xi^{-}$. The differential and total cross sections were determined 


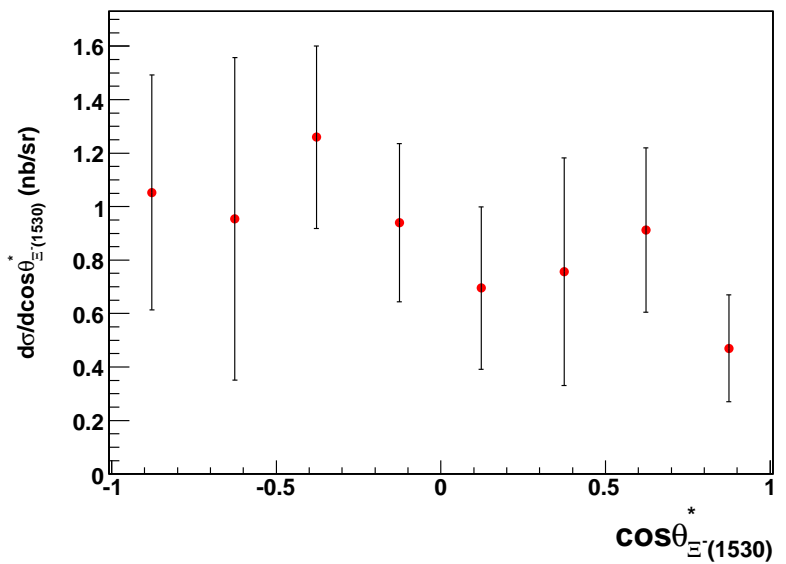

Fig. 3. Differential cross sections for the $\Xi^{-}(1530)$ in the photon energy range of 3.354.75 GeV. Both statistical and systematic uncertainties are included.

for photon beam energies from 2.75 to $4.75 \mathrm{GeV}$. The $\Xi^{-}$angular distributions, as well as the $K^{+} \Xi^{-}$invariant mass spectra, are consistent with a possible production mechanism of $Y^{*} \rightarrow \Xi^{-} K^{+}$through a $t$-channel process (see Ref. 3 for details). The integrated cross section, showing the rise from threshold, is shown in Fig. 2.

The $\Xi^{-}(1530)$ is also clearly present in the spectrum of Fig. 1, with about 700 events. The differential cross sections of the $\Xi^{-}(1530)$ was measured for the first time in photoproduction through the reaction $\gamma p \rightarrow$ $K^{+} K^{+}(X)$ for photon beam energies from 3.35 to $4.75 \mathrm{GeV}$. The differential cross section is shown in Fig. 3. The background shape was determined from events with an additional $K^{-}$detected, since the background is dominated by reactions where one of the kaons was actually a misidentified pion.

The reaction $\gamma p \rightarrow K^{+} K^{+} \pi^{-}\left(\Xi^{0}\right)$ was also investigated to search for excited cascade resonances decaying to $\pi^{-} \Xi^{0}$. However, no significant signal of excited cascade states other than the $\Xi^{*-}(1530)$ is observed.

\section{Search for cascade pentaquarks}

Narrow bound states of four quarks and one anti-quark have been the focus of intense searches since the report by the LEPS collaboration ${ }^{5}$ of a baryon with $S=+1$, called the $\Theta^{+}$, with a mass of $1.54 \mathrm{GeV}$. This state with 


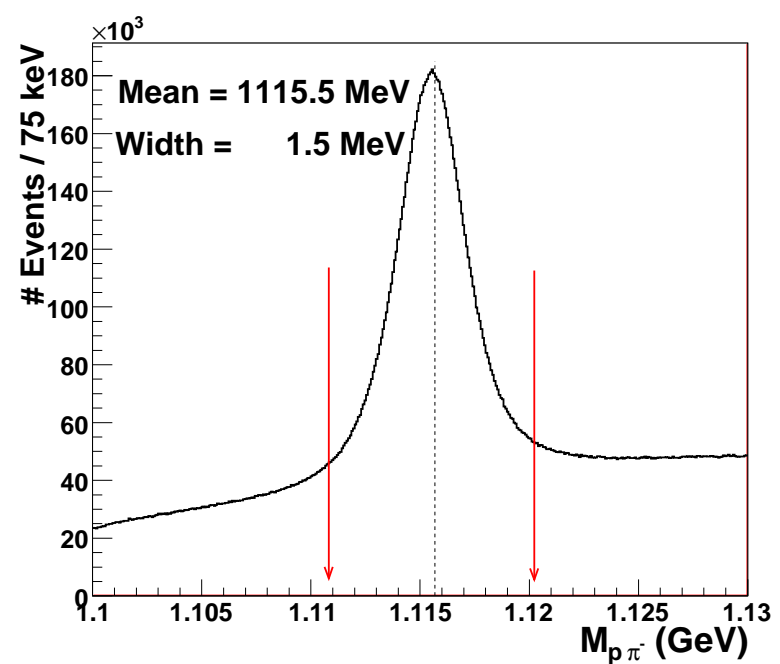

Fig. 4. Invariant mass of $p \pi^{-}$, showing the reconstructed $\Lambda \mathrm{s}$ in the data sample. The arrows show the selection of the $\Lambda$ interval.

"exotic" quantum numbers, was identified as a member of the anti-decuplet of baryons. For a review of the experimental situation consult Ref. 6 . The two other exotic states in the anti-decuplet have $S=-2$, one with charge $Q=-2$ and the other with $Q=+1$. They are cascades states with $J=\frac{3}{2}$, in contrast to the normal octet cascades states which have $J=\frac{1}{2}$. The Particle Data Group has assigned the name of $\Phi(1860)$ to this state, where the mass of $1.862 \mathrm{GeV}$ is taken from the single experimental report by the NA49 collaboration. ${ }^{7}$ This measurement was conducted with $\mathrm{p}+\mathrm{p}$ collisions at a center-of-mass energy $\sqrt{s}=17.2 \mathrm{GeV}$. The two exotic states are $\Phi^{--}$and the $\Phi^{+}$, and their partners are denoted $\Phi^{-}$and $\Phi^{0}$. The NA49 collaboration reported evidence for the exotic pentaquark $\Phi^{--}$and the $\Phi^{0}$, which were reconstructed from their decay products from $\Phi^{--} \rightarrow \Xi^{-} \pi^{-}$ and $\Phi^{0} \rightarrow \Xi^{-} \pi^{+}$. We note that many experiments in a variety of conditions (see Ref. 8 and references therein) have not been able to confirm this initial observation.

Guidance for where and how to search for cascade pentaquarks is very sparse. Within the quark models, the mass scale for the $\Theta^{+}$can be estimated to be about the mass of the nucleon plus the mass of the kaon. But most models have taken the mass scale from experimental reports. Predictions for the masses of the cascade pentaquarks range from 1.75 to $2.07 \mathrm{GeV}$. The width of the $\Phi(1860)$ is estimated to have several times the 


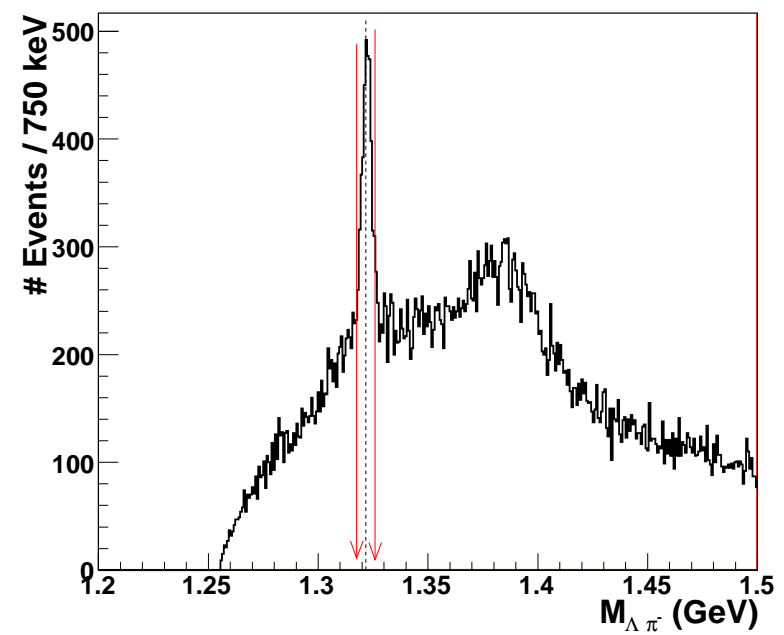

Fig. 5. Invariant mass of $p \pi^{-} \pi^{-}$, with approximately 1700 reconstructed $\Xi^{-}$. The arrows show the selection of the $\Xi$ interval. The broad peak to the right corresponds to the production of the $\Sigma(1385)$.

width of the $\Theta^{+}$. There is only one calculation of the production cross section of exotic cascades with photon beams. ${ }^{9}$ The calculated production off the neutron is larger than off the proton, but uncertainties are very large. At $E_{\gamma}=5 \mathrm{GeV}$ the estimated $\sigma\left(\gamma n \rightarrow K^{+} K^{+} \Xi^{--}\right)$including effective form factors varies between 0.4 and $1.5 \mathrm{nb}$ depending on the value of the

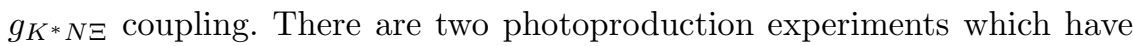
set limits on the production of the $\Phi^{--}$. The FOCUS experiment, ${ }^{8}$ with a untagged bremsstrahlung beam produced with $300 \mathrm{GeV}$ electrons, has set the limit of $\sigma\left(\Phi^{--}\right) \times \mathrm{BR} / \sigma\left(\Xi^{*}(1530)\right)<0.032-0.091(95 \% \mathrm{CL})$. The HERMES experiment, ${ }^{10}$ using a $27 \mathrm{GeV}$ positron beam, has set a limit of $\sigma\left(\Phi^{--}\right) \times \mathrm{BR}<1.0-2.1 \mathrm{nb}(90 \% \mathrm{CL})$. The reported ranges reflect different assumptions used for extracting the limits.

\section{Experiment eg3}

The eg3 experiment used a tagged bremsstrahlung photon beam in the experimental Hall B at Jefferson Lab. The purpose of this specific analysis is to search for the $\Phi^{--}$in a photoproduction experiment with the CLAS detector. $^{2}$ The electron beam energy was $5.77 \mathrm{GeV}$. Data were taken at a nominal current of $30 \mathrm{nA}$ and used a $5 \times 10^{-4}$ r.l. radiator to produce the photon beam. The photon beam was incident on a $40-\mathrm{cm}$ long and $4-\mathrm{cm}$ 


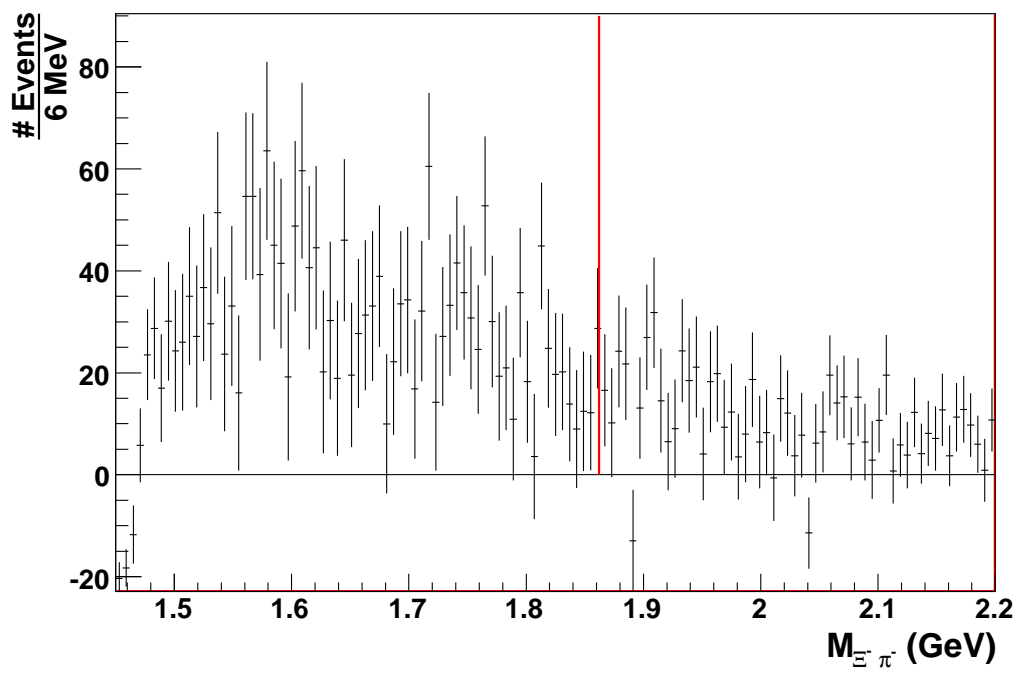

Fig. 6. Invariant mass of $\Xi^{-} \pi^{-}$, showing a featureless spectrum. The mass of the single reported observation at $1.86 \mathrm{GeV}$ is indicated by the vertical line.

diameter liquid deuterium target positioned $50 \mathrm{~cm}$ upstream of the nominal CLAS target position. The data were registered using a Level 1 trigger, which consisted of two configurations. The primary trigger configuration required at least three tracks in three different sectors. The second trigger configuration, prescaled, required only two tracks in two sectors and was used for systematic studies. Both triggers required a coincident photon in the energy range between 4.5 and $5.5 \mathrm{GeV}$. The experiment ran with a typical live time of greater than $80 \%$.

We describe the analysis to look for a signal in the reaction $\gamma d \rightarrow \Phi^{--} X$ via the decay sequence $\Phi^{--} \rightarrow \pi^{-} \Xi^{-}(1321) \rightarrow \pi^{-}\left(\pi^{-} \Lambda\right) \rightarrow \pi^{-} \pi^{-}\left(\pi^{-} p\right)$. The analysis required four reconstructed tracks in the drift chambers, one positive and three negative. The time-of-flight from the target to the scintillator planes (approximately $5 \mathrm{~m}$ ) identified the positive track as a proton, and the negative tracks as pions. Fig. 4 shows the invariant mass of the the $p \pi^{-}$pair most likely to be the product of $\Lambda$ decays. A very clean peak is found with a width of $1.5 \mathrm{MeV}$. A second $\pi^{-}$was used in combination with the $\Lambda$ to reconstruct the $\Xi^{-}$, with the decay sequence of the $\Lambda$ and $\Xi$ used to increase the signal-to-background (Fig. 5). Finally the invariant mass of the third $\pi^{-}$and the events in the $\Xi^{-}$peak, with side-bands subtracted, is shown in Fig. 6.

The spectrum in Fig. 6 was examined over the mass range from 1.5 to 
2.2 GeV for a narrow resonance structure with a width comparable to our experimental resolution of $10 \mathrm{MeV}$. No signal is found in the $\Xi^{-} \pi^{-}$mass spectrum. At the mass of the NA49 report of $1.86 \mathrm{GeV}$, we set a preliminary upper limit for the inclusive production of $\Xi^{--}$of less than $1 \mathrm{nb}$ (90\% CL).

\section{Acknowledgments}

This work was supported by the U.S. Department of Energy contract DEAC05-06OR23177, under which Jefferson Science Associates, LLC operates the Thomas Jefferson National Accelerator Facility. I would like to especially thank L. Guo, for discussions regarding the cascade cross section measurements, and my colleagues from eg3, the search for cascade pentaquarks.

\section{References}

1. W. M. Yao et al., J. Phys. G33, 1 (2006).

2. B. A. Mecking et al., Nucl. Instrum. Meth. A503, 513 (2003).

3. L. Guo et al., Phys. Rev. C76, p. 025208 (2007).

4. K. Nakayama, Y. Oh and H. Haberzettl, Phys. Rev. C74, p. 035205 (2006).

5. T. Nakano et al., Phys. Rev. Lett. 91, p. 012002 (2003).

6. K. H. Hicks, Prog. Part. Nucl. Phys. 55, 647 (2005).

7. C. Alt et al. (NA49 Collaboration), Phys. Rev. Lett. 92, p. 042003 (2004).

8. K. Stenson, Int. J. Mod. Phys. A20, 3745 (2005).

9. W. Liu and C. Ko, Phys. Rev. C 69, p. 045204 (2004).

10. A. Airapetian et al., Phys. Rev. D71, p. 032004 (2005). 